\title{
PERFORMANCE OF SOFT-OUTPUT EQUALIZATION AND CONVOLUTIONAL CODING OVER FREQUENCY-SELECTIVE FADING RADIO CHANNELS
}

\author{
Antonio Valdovinos, Antonio Navas, Antoni Gelonch, Fernando J. Casadevall \\ Department of Signal Theory and Communications \\ E.T.S. Ingenieros de Telecomunicación \\ Universidad Politécnica de Cataluña \\ Apdo. 30002, 08080 Barcelona, SPAIN \\ Tel. $3434015948 \quad$ Fax. 3434017200 \\ e-mail.toni@xaloc.upc.es
}

\begin{abstract}
This paper presents an analysis of soft-output equalization and codification techniques applied to 4 and 16-QAM TDMA radio systems for frequency selective fading channels. In particular, Typical Urban (TU) and Hilly Terrain (HT) environments have been considered. The use of the soft information provided by the equalizer enhances the system performance considerably. Specifically, an improvement of more than $4 \mathrm{~dB}$ can be achieved in relation to hard decoding techniques. The necessity of introducing some interleaving in order to destroy the channel memory has also been stated. The actual investigated equalizers have been a Soft-Output Decision-Feedback equalizer (SODFE) and a SoftDecision M-Algorithm (SDMA) jointly with convolutional coding and both ideal and finite interleaving.
\end{abstract}

\section{INTRODUCTION}

The growing importance of wireless digital radio systems has led to the development of high-performance techniques necessary to compensate for the severe frequency-selective fading introduced by the channel. In order to combat the Intersymbol interference caused by the multipath propagation, the use of equalization techniques becomes mandatory. From the point of view of system performance, non-linear equalizers are more efficient than linear ones since they can compensate for deeper in-band nulls due to selective fading. Specifically, Decision-Feedback Equalizers (DFE) and the Maximum Likelihood Sequence Estimation (MLSE) and Maximum a Posteriori (MAP) algorithms can be considered [1]. If the channel memory is too long, the probabilistic equalizers, that is MLSE and MAP, become impractical due to their computational complexity, which grows exponentially with the channel memory. Therefore, simplified algorithms have to be introduced in spite of a moderate loss in bit error rate (BER) performance. The most widely introduced techniques are the M-Algorithm (MA), [2], and the SDMA, [3], which have proved to be appropriate for reliable operation with relatively low computational cost. However, the only use of equalization techniques does not usually provide good enough performance to operate in practical systems and some coding and interleaving which destroys the channel memory are required. On the other hand, the decoder usually operates on the hard decisions made by the demodulator, which leads to an information and, consequently, to a performance loss. This drawback can be circumvented by making use of the additional information contained in the unquantized samples given by the demodulator.

In this paper both DFE and SDMA techniques have been investigated jointly with convolutional coding and ideal and finite interleaving in two realistic environments, namely Typical Urban (TU) and Hilly Terrain (HT). The chosen modulation schemes have been M-QAM patterns because they are spectrum efficient and so, appropriate for environments where a high density of traffic is generated. In particular, 4 and 16-QAM modulation schemes have been considered. Performance results, in terms of bit error rate values, obtained by means of computer simulation are presented.

\section{SYSTEM DESCRIPTION}

The block diagram of the simulated transmission system is shown in fig.1. The output sequence from the source is protected by a convolutional code and passed to an interleaver with some finite span and depth. The interleaved sequence is M-QAM modulated and transmitted to the channel after being passed through the transmitter filter, $h_{T}(t)$, which is a root-raised cosine filter with a roll-off factor equal to 0.5 . Therefore, the lowpass 


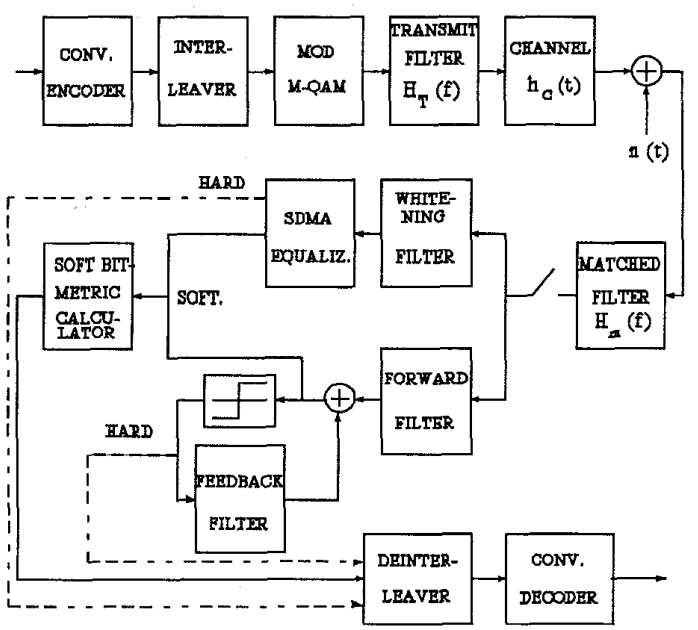

Figure 1. Lowpass equivalent model of the transmission system.

equivalent M-QAM signal transmitted to the channel can be expressed as:

$$
x(t)=\sum_{k=-\infty}^{\infty} a_{k} \cdot h_{T}(t-k T)+j b_{k} \cdot h_{T}(t-k T)
$$

where $\left\{a_{k}\right\}$ and $\left\{b_{k}\right\}$ are independent data sequences of duration $\mathrm{T}$ for the in-phase and quadrature channels respectively. They are selected from the set $\left\{ \pm 1, \pm 3, \ldots, \pm\left(M^{1 / 2}-1\right)\right\}$ with $M=4$ for $4-Q A M$ and $M=16$ for 16-QAM.

At the receiver a matched filter $H_{m}(f)$ is incorporated, then:

$$
h_{m}(t)=F^{-1}\left[H_{m}(f)\right]=h_{1}^{+}(-t)
$$

where + denotes the conjugate, $\mathrm{F}^{-1}$ is the inverse Fourier transform and $h_{1}(t)$ is the equivalent impulse response prior to the matched filter:

$$
h_{1}(t)=h_{T}(t) * h_{c}(t)
$$

Therefore, the global impulse response results:

$$
h(t)=h_{T}(t) * h_{c}(t) * h_{m}(t)=h_{1}(t) * h_{1}^{+}(-t)=h^{+}(-t)
$$

Let us notice as the receiver filter is matched not only to the root-raised cosine transmitting filter but also to the entire impulse response $h_{1}(t)$, which is affected by the channel impulse response variations, and thus needs to be continually updated. It could be implemented in accordance with a FIR structure from the estimated impulse response. In this study, a ideal channel estimation is assumed and, so, the ideal matched filter is always available. The function $h_{c}(t)$ models the channel behavior that introduces selective fading in the radio link. The channel is assumed to be wide-sense stationary uncorrelated scattering (WSSUS) and it is represented by a unique correlation function referred to as the Power Delay Profile, $\mathrm{P}(\mathrm{t})$, and given by:

$$
P(t)=\left\langle/\left.h_{c}(t)\right|^{2}\right\rangle
$$

The multipath phenomenon can be described in terms of time delays and their associated power values. No Doppler shifts have been considered. The models adopted correspond to those proposed by COST-207, [4], to describe typical urban (non-hilly) and hilly terrain environments and is based on representing the channel by a finite number of taps, each one determined by its time delay and average power. The amplitude of each tap is Rayleigh distributed. This models is described by a continuous power delay profile and approximated through a discrete number of taps, [4]. At the output of the channel model, AWGN with a double-sided power spectral density $\mathrm{N}_{0} / 2$ is added.

The signal at the matched filter output is expressed as:

$$
\begin{gathered}
r(t)=\sum_{k=-\infty}^{\infty}\left[a_{k} \cdot h^{R}(t-k T)-b_{k} \cdot h^{I}(t-k T)\right]+ \\
+j \sum_{k=-\infty}^{\infty}\left[b_{k} \cdot h^{R}(t-k T)+a_{k} \cdot h^{I}(t-k T)\right]+n_{x}(t)+j n_{y}(t)
\end{gathered}
$$

where

$$
h(t)=h^{R}+j h^{l}=\left[F^{-1}\left[H_{\imath}(f) \cdot H_{m}(f)\right] * h_{c}(t)\right] . G
$$

with $*$ the convolution operator. $\mathrm{G}$ is a gain factor that ensures a constant average power at the demodulator input. It is introduced in order to consider the presence of automatic gain control (AGC) and is only necessary for 16-QAM patterns since in 4-QAM we have zero-crossing decision boundaries.

After the matched filter, we have considered two possible branches corresponding to the SDMA and SODFE equalizers. For the first one, the global impulse response prior the SDMA equalizer is required to be $\mathrm{h}(\mathrm{k})$ 
$=0$ for $\mathrm{k}<0$ and so, a whitening filter (WF) is necessary. This filter has been constructed as a DFE forward filter whose taps are adjusted following the minimum meansquare error (MMSE) technique because even though ideally the WF shoud be adjusted by means of the zero forzing criterion [5], unavoidably the number of taps is finite and, therefore, the residual ISI effect is not negligible. Furthermore, the SDMA must work with a finite impulse response length, and so $h(k)=0$ for $k>L$. We should take a value of $\mathrm{L}$ high enough to consider all the significant ISI components, but not so high that the computational complexity becomes unreasonable. The detailed SDMA algorithm can be found in [3]. On the other hand, a DFE structure with symbol period (T) spacing between stages has also been investigated. The MMSE technique has also been adopted to calculate the tap values. In this case, the soft output is obtained from the unquantized signal. Both equalizers deliver soft decisions for each symbol and, from them, the respective bit metrics are calculated [3][6]. Finally, after deinterleaving, these metrics are fed to the decoder to exploit the soft information. In order to compare soft versus hard-decision performance, we have also considered the output sequence from the quantizer which is fed directly to the de-interleaver.

\section{RESULTS}

The performance of soft-output equalization and convolutional coding techniques in outdoor radio channels have been investigated using the bit error rate (BER) as quality criterion. The signal-to noise ratio is given by:

$$
S N R=\frac{E_{b}}{N_{o}}+10 \log M
$$

where $E_{b}$ is the average bit energy and $M=4$ and $M=16$ for 4 and 16-QAM respectively.

We have considered bit rate values of $1 \mathrm{Mbit} / \mathrm{s}$ and $200 \mathrm{Kbit} / \mathrm{s}$ for TU and HT environments since they lead to the same normalized delay spread defined as the product $\tau . R_{b}$, where $R_{b}$ is the bit rate and $\tau$ is the root mean-square delay spread, which is a mesure of the width of the Power Delay Profile and is equal to $1 \mu$ s for TU and $5 \mu$ s for HT in the analyzed models[4].

The number of taps for the SODFE has been chosen 6 for the forward and 5 for the feedback filters.

For the SDMA equalizer a channel memory length $L=6$

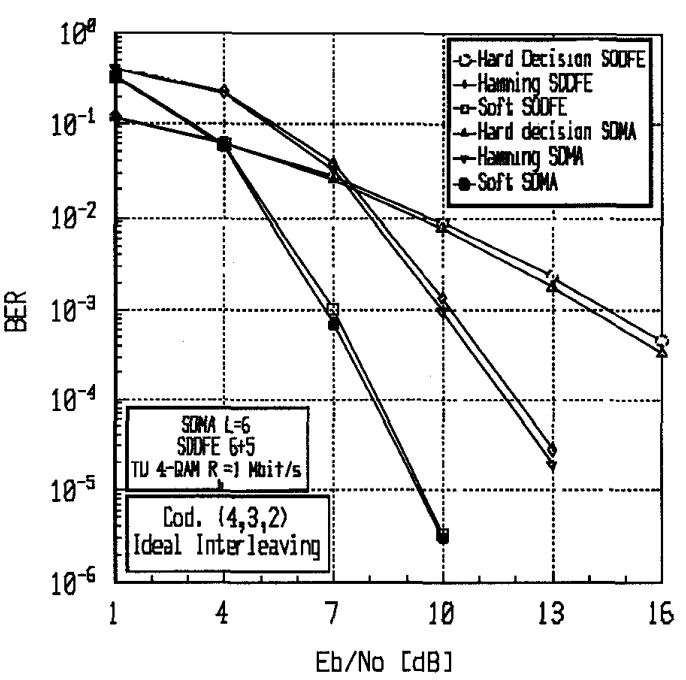

Figure 2. BER versus $\mathrm{E}_{\mathrm{b}} / \mathrm{N}_{\mathrm{o}}$.TU and 4-QAM.

has been considered. Various techniques have been compared. In particular, hard-decision versus soft-decision has been investigated.

In fig. 2 the evolution of the BER as a function of $E_{b} / N_{o}$ for 4-QAM and TU environments is depicted. A convolutional code with rate $r=3 / 4$ and constraint length of $\mathrm{K}=2$ is used to protect the source data. We can observe that there is not appreciable difference between the SODFE and SDMA structures, not more than $0.5 \mathrm{~dB}$, although the latter is always sligthly better. However, the performance improvement obtained when introducing decoding techniques is very important, particularly when Soft metrics are considered. For a BER of $10^{-2}$ Soft metrics offer an improvement of $3 \mathrm{~dB}$ with reference to hard decoding and $4.5 \mathrm{~dB}$ with reference to no coding. For increasing values of $\mathrm{E}_{\mathrm{b}} / \mathrm{N}_{\mathrm{o}}$ we can notice that the difference between both soft and hard decoding techniques varies slowly and is of about $3 \mathrm{~dB}$ for $10^{-3}$ and $4 \mathrm{~dB}$ for $10^{-4}$. In contrast, the relative degradation if no coding is considered increases for higher $E_{b} / N_{o}$ values. For a BER of $10^{-3}$ this degradation is $7.5 \mathrm{~dB}$ and for $10^{-4}$ could be $10.5 \mathrm{~dB}$ with reference to Soft metrics. A similar comparison can be carried out from the observation of fig. 3 , which represents the BER evolution for HT environments and 4-QAM modulation schemes. In this case, to achieve a BER of $10^{-3}$ the difference between soft and hard decoding is about $4 \mathrm{~dB}$. It must be born in mind that the delay spread introduced by the channel in HT environments is five times greater than TU channels, and so, although figures 2 and 3 present similar BER values for equal $E_{b} / N_{0}$, in the latter the bit rate is five 


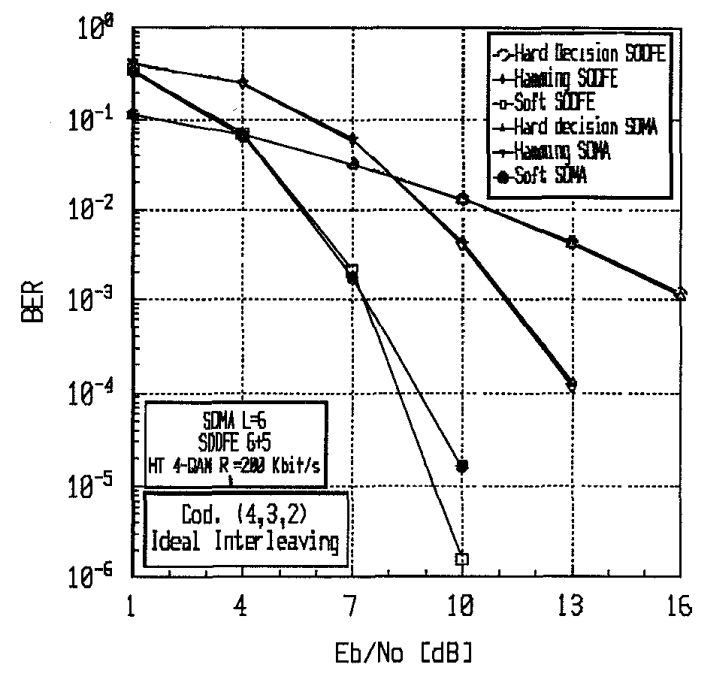

Figure 3. BER versus $\mathrm{E}_{n} / \mathrm{N}_{0}$. $\mathrm{HT}$ and 4-QAM.

times lower.

Fig. 4 shows the system performance for $16-$ QAM and TU environments. In this case the difference between SODFE and SDMA is slightly higher, but never more than $1 \mathrm{~dB}$. From this figure we can notice that the behavior is similar to that of 4-QAM systems, but it is clear that the $\mathrm{E}_{b} / \mathrm{N}_{\mathrm{o}}$ value needed to guarantee a certain value of BER is considerably higher. Actually, a 16-QAM system needs approximately $6 \mathrm{~dB}$ more than 4-QAM to achieve the same performance. Therefore, higher level modulation schemes are useful to increase the spectral efficiency, but not the maximum bit rate reached by the system due to its higher sensitivity to ISI effects.

Fig. 5 presents the effect of considering finite instead of infinite interleaving. We have focused on TU environments, 4-QAM and Soft metrics. If no interleaving at all is introduced, the degradation due to the channel memory is as high as $4 \mathrm{~dB}$ for BER $=10^{-2}, 6 \mathrm{~dB}$ for $\mathrm{BER}=10^{-3}$ and $8 \mathrm{~dB}$ for $\mathrm{BER}=10^{-4}$. If a finite interleaving depth of 4 is considered, the degradation is 2,3 and $4 \mathrm{~dB}$ respectively for SDMA equalization. In the case of SODFE an additional degradation of $1 \mathrm{~dB}$ isrevealed. From this figure we can conclude that some kind of interleaving is needed in order to take the most advantage from the soft information delivered by the equalizer. Otherwise the effect of coding not only is not advantageous, but may introduce a higher performance degradation.

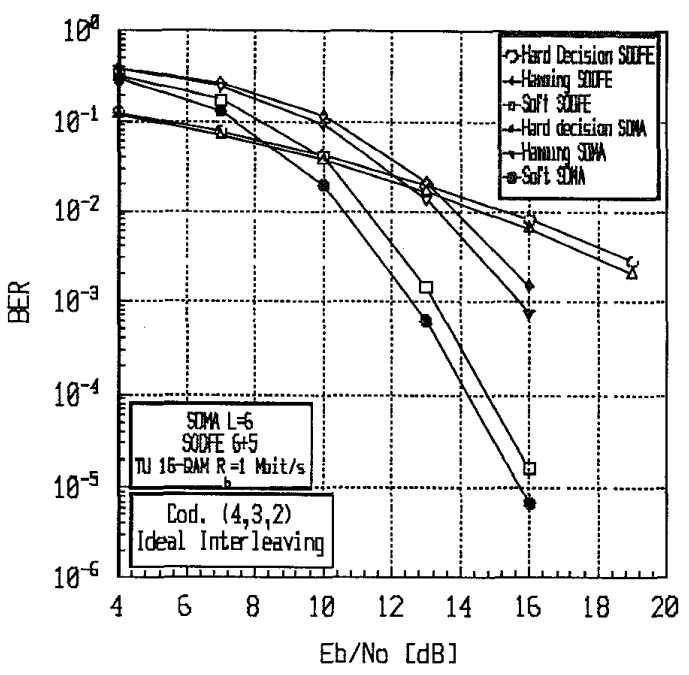

Figure 4. BER versus $\mathrm{E}_{\mathrm{b}} / \mathrm{N}_{\mathrm{o}}$.TU and 16-QAM.

Finally, we have analyzed the effect of varying the coder rate from $r=3 / 4$ to $2 / 3$ and $1 / 2$ when an interleving depth of 4 is considered. The results are presented in fig. 6 . It can be noticed that passing from $r=3 / 4$ to $2 / 3$ an improvement of about $2 \mathrm{~dB}$ is achieved, and roughly $2.5 \mathrm{~dB}$ when passing from $2 / 3$ to $1 / 2$. This may not seem a great improvement taking into account the increasing bandwidth required when $r$ decreases.

\section{CONCLUSIONS}

In this paper we have investigated the performance of equalization and codification techniques applied to 4 and 16-QAM TDMA radio systems in two typical outdoor environments where the dispersive nature of the channel introduces both intersymbol interference and Rayleigh fluctuations of the signal-to-noise ratio. The use of equalization techniques which provide soft information to the decoder, namely SODFE and SDMA, has proved to be very effective and leads to BER values considerably lower than those achieved by using the more conventional hard-decision techniques. Moreover, we have presented a brief comparison between 4 and 16-QAM modulation schemes. From the results obtained we can conclude that the use of high-order QAM patterns does not offer any advantage in terms of maximum bit rate achievable by the system, and its use is only advisable when a high spectral efficiency is required. We have also stated the necessity of introducing some finite interleaving, as high as the data delay requirements make 


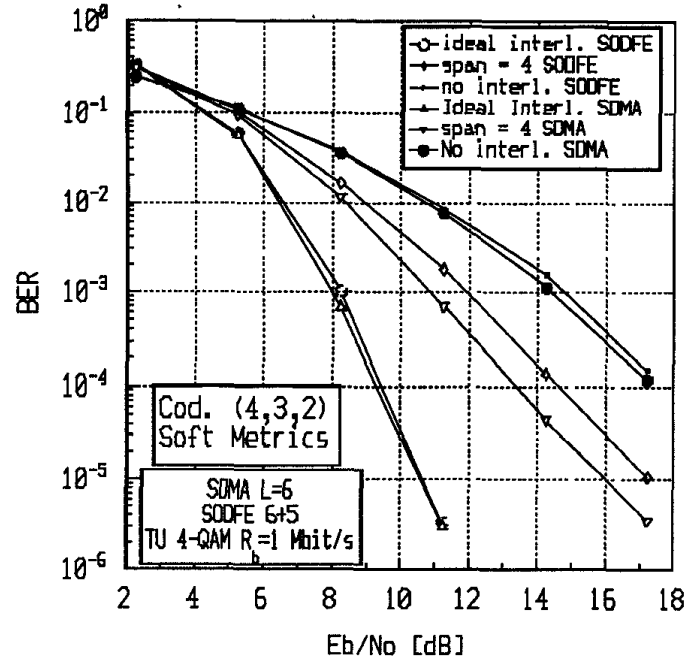

Figure 5. BER versus $\mathrm{E}_{\mathrm{o}} / \mathrm{N}_{\mathrm{o}}$. TU and 4-QAM.

possible, in order to destroy the channel memory, and we have evaluate the results for three different cases. Finally, the effect of varying the code rate, $r$, has been analyzed, but, obviously, the tradeoff between BER and bandwidth has to be solved in each particular case.

\section{REFERENCES}

J.G. Proakis. "Adaptive Equalization for TDMA Digital Mobile Radio". IEEE Trans. on Vehic. Tech. Vol. 40. NO 2. May 1991. pp. 333-341.

[2] A. Baier and G. Heinrich. "Performance of MAlgorithm MLSE Equalizer in FrequencySelective Fading Mobile Radio Channels". Proc. IEEE ICC'89, Boston, MA, 1989, pp. 281-285.

[3] R. Mehlan and H. Meyr. "Soft Output MAlgorithm Equalizer and Trellis-Coded Modulation for Mobile Radio Communications". Proc. 42nd IEEE Vehic. Technol. Conf., Denver, CO, 1992, pp. 586,591.

[4] COST 207: "Digital Land Mobile Radio Communications". Final Report. Published by the Commission of the European Communities.Luxembourg, 1989. pp. 135-147.

[5] E.A. Lee and D.G. Messerschmitt. "Digital Communications". Kluwer Academic Publishers. 1988. pp. 345-346.

[6] Y.J. Liu, M. Wallace and J.W. Ketchum. "A Soft-Output Bidirectional Decision Feedback Equalization Technique for TDMA Cellular

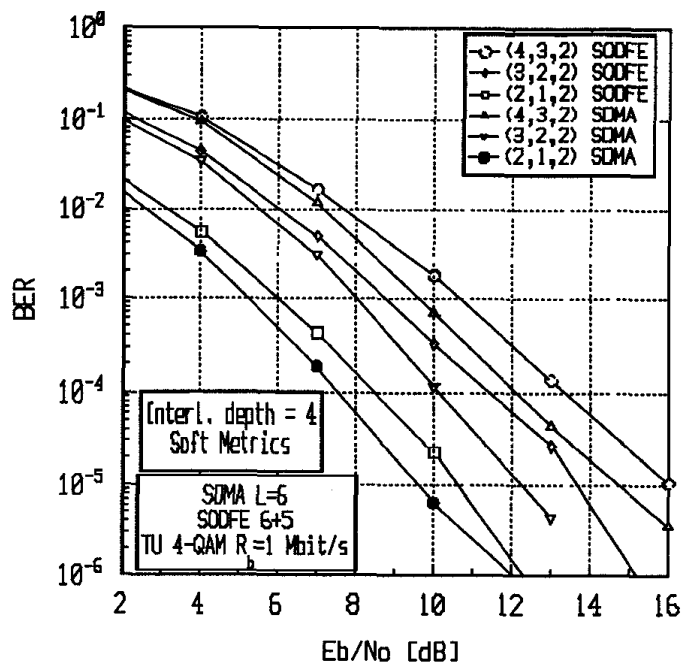

Figure 6. BER versus $\mathrm{E}_{\mathrm{b}} / \mathrm{N}_{\mathrm{o}}$. TU and 4-QAM.

Radio". IEEE Journal on Selected Areas in Comm.. Vol 11. NO. 7. September 1993. pp. 1034-1045. 